\title{
Ovarian hyper stimulation syndrome in a spontaneous singleton pregnancy: a case report
}

\author{
Anuj Kumar Sharma ${ }^{1 *}$, Radha Rastogi ${ }^{1}$, Kalpesh Patel ${ }^{1}$, K. G. Vivek ${ }^{2}$ \\ ${ }^{1}$ Department of Obstetrics and Gynaecology, RNT Medical College, Udaipur, Rajasthan, India \\ ${ }^{2}$ Department of Obstetrics and Gynaecology, Base Hospital Barrackpore, West Bengal, India
}

Received: 22 December 2021

Revised: 18 January 2022

Accepted: 19 January 2022

\section{*Correspondence:}

Dr. Anuj Kumar Sharma,

E-mail: dranujsharma.gynac@gmail.com

Copyright: () the author(s), publisher and licensee Medip Academy. This is an open-access article distributed under the terms of the Creative Commons Attribution Non-Commercial License, which permits unrestricted non-commercial use, distribution, and reproduction in any medium, provided the original work is properly cited.

\begin{abstract}
Ovarian hyper stimulation syndrome (OHSS) is extremely rare in spontaneous pregnancies. Spontaneous OHSS can result from glycoprotein hormones stimulating follicle-stimulating hormone receptors (FSHR). Our case reinforces the importance of a prompt diagnosis and management in all pregnant patients presenting with acute abdomen and ovarian masses. We report a case of spontaneous singleton pregnancy at 12-week POG presented with abdominal distension and enlarged ovaries. Patient was successfully managed with supportive treatment comprise of intravenous (IV) Albumin, thromboprophylaxis, dopamine agonist and insulin sensitizer. Spontaneous OHSS should be included in the differential diagnosis of acute abdomen in pregnant women. Since spontaneous OHSS can be associated with lifethreatening complications, it requires early diagnosis for successful management. The etiology should be determined in order to focus the treatment and avoid future complications.
\end{abstract}

Keywords: Secondary OHSS, Thromboprophylaxis, hCG, GnRH analogues, Dopamine agonist

\section{INTRODUCTION}

Ovarian hyper stimulation syndrome (OHSS), an iatrogenic and a dreadful complication of controlled ovarian stimulation during Assisted reproductive technology (ART) occurs as an exaggerated response to exogenous gonadotropins and rarely to ovulation inducing agents like clomiphene citrate or due to spontaneous conception. Extremely rare in spontaneous pregnancies, incident is less than $1 \%$. This self-limiting disorder characterised by various clinical manifestations due to increased capillary permeability and accumulation of fluid in extravascular space can have a downhill course and prove to be fatal if not timely recognised and intervened. ${ }^{1}$

OHSS is characterized by enlargement of the ovaries and the formation of multiple cysts along with collection of fluid in peritoneal cavity. In contrast with iatrogenic forms, which typically appear earlier, spontaneous OHSS usually develops between the 8th and 14th weeks of gestation; it is sometimes familial and recurrent. ${ }^{2}$

Follicle-stimulating hormone (FSH) receptor could be stimulated by high levels of FSH or other glycoprotein hormones with identical beta subunit, such as thyroidstimulating hormone (TSH), luteinizing hormone ( $\mathrm{LH})$, and chorionic gonadotrophin hormone (hCG). Mutations of the FSH receptor (FSHR) gene that make the receptors more sensitive may also be possible.

Risk factors associated with OHSS can be divided in primary and secondary risk factor. Factors present before stimulation and likely to amplify the response to ovarian stimulation are primary risk factors. They include: young age, low BMI, a history of elevated response to 
gonadotrophins, previous OHSS, polycystic ovary syndrome (PCOS), a high serum anti Mullerian hormone level. ${ }^{3}$ Secondary risk factors are the ones which become obvious during the course of stimulation; i.e. absolute levels or rate of increase of serum estradiol (E2), follicular size and number, and number of oocytes collected. ${ }^{4}$

\section{CASE REPORT}

This was a 25-year-old G2 P1 L1 woman, with a spontaneous pregnancy at 12 weeks of gestational age according to the last menstrual period. The pregnancy was uneventful until 11 weeks of gestational age. After that, the woman started complaining of progressive abdominal distension with abdominal discomfort. Her menstrual cycles were irregular (45-60 days cycle) without dysmenorrhoea, acne or hirsutism. There was no history of any known gynaecological diseases.

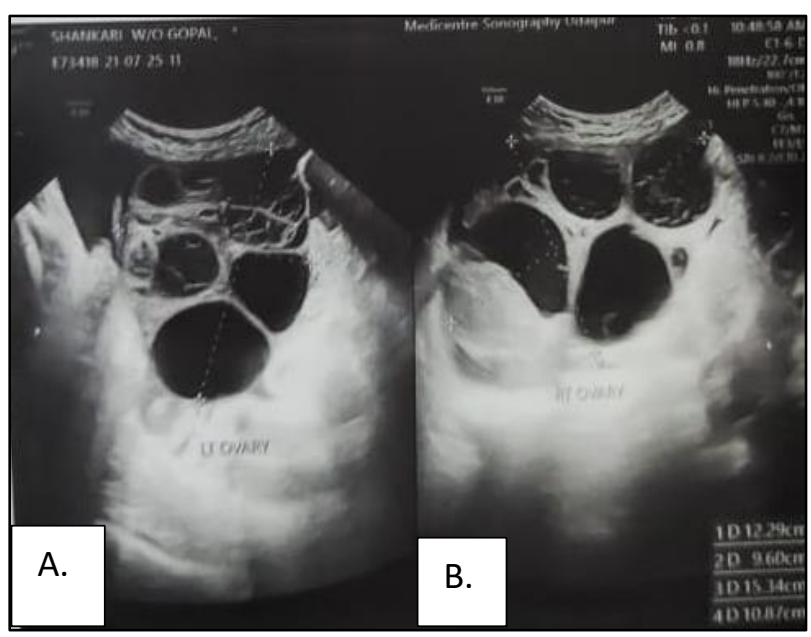

Figure 1: (A) and (B) USG Findings of uterus and B/L Ovaries (revealed a single live fetus and normal amniotic fluid. B/L ovaries were enlarged with multiple cysts).

On admission, the patient was hemodynamically stable. Her pelvic examination showed uterus size compatible with gestational age, and large and mobile bilateral adnexal masses. Laboratorial exams showed haemoglobin level of $5.9 \mathrm{~g} / \mathrm{dL}$, hematocrit of $17.2 \%$, hypoalbuminemia of $2.3 \mathrm{~g} / \mathrm{dL}$, and hypoproteinaemia of $5.1 \mathrm{~g} / \mathrm{dL}$; coagulation tests, hepatic and renal function were within normal range (urea of $17 \mathrm{mg} / \mathrm{dL}$ and creatinine of 0.6 $\mathrm{mg} / \mathrm{dL}$ ). Serum b hCG was $152410 \mathrm{mIU} / \mathrm{ml}$ and thyroidstimulating hormone (TSH) was $2.2 \mu \mathrm{UI} / \mathrm{ml}$. Sonography revealed a single live fetus at 12 weeks 2 days CRL and normal amniotic fluid. The patient's ovaries were enlarged (right ovary was $15.3 \mathrm{~cm}$ and the left ovary was $12.4 \mathrm{~cm}$ measured from their major axis) with multiple cysts, and moderate amount of peritoneal fluid (Figure 1 (A) and (B)).

During hospitalization, she was under clinical and sonographic surveillance and received 2 units of PRBC, intravascular human albumin for 3 days, IV fluids, prophylactic dose of enoxaparin, prophylactic IV Antibiotics, oral low dose aspirin, Cabargolin $0.5 \mathrm{mg}$ alt day and Metformin $500 \mathrm{mg}$ twice a day. On day-2 of her admission, she found to have missed abortion and aborted subsequently. She remained clinically stable, and recovery was seen in lab reports and sonographic parameters. The patient was discharged on the sixth day of hospitalization. At hospital discharge, the ultrasound showed large ovaries, the right with $8.4 \mathrm{~cm}$ and the left with 6.2 with minimal ascites. On discharge, patient was given oral contraceptives and oral haematinics for 6 months.

\section{DISCUSSION}

Spontaneous forms of OHSS are extremely rare and develop between 8 and 14 weeks of amenorrhoea, which differ from iatrogenic OHSS that usually start between 35 weeks of amenorrhoea. It is generally associated with either abnormally high values of hCG or high levels of TSH, which could stimulate the ovaries.

The exact etio-pathological mechanism of OHSS is still elusive but the culprit molecule for its initiation has been documented to be human chorionic gonadotropin (hCG) as the syndrome does not develop if hCG is withheld. VEGF, known as the 'vascular permeability factor' also has been found to play the most critical role. VEGF is the important mediator of hCG-dependent ovarian angiogenesis. VEGF is expressed in human ovaries and it has been observed that VEGF mRNA levels increases after hCG administration in granulosa cells and VEGF levels correlate with OHSS severity. The hallmark mechanism postulated for the presentation of this syndrome is increased vascular permeability and extravasations of fluid from the intravascular compartment to the third space compartment which in turn causes hemoconcentration with reduced organ perfusion, alterations in blood coagulation and leakage of fluid into the peritoneal cavity and lungs.

Based on the symptoms and associated features the disease process is classified by its severity into mild, moderate, severe and critical.

De Leener classified spontaneous OHSS syndrome into three types based on clinical presentation and FSH receptor mutation. ${ }^{5}$ Type $\mathrm{I}$ is attributed to the mutated FSH receptor and seems to cause recurrent spontaneous OHSS. Type II is secondary to high levels of hCG as in hydatiform mole and multiple gestation and it is the most common one. Type III is associated with hypothyroidism.

The treatment of OHSS has been primarily empirical and prevention has formed the mainstay of management. The preventive strategies aim, to target women at high risk and institution of various pharmacological and nonpharmacological interventions on them. The various preventive strategies are: 


\section{Type of protocol}

Multiple studies have demonstrated that stimulation protocols utilising gonadotropin releasing hormone (GnRH) antagonists for ovarian suppression are associated with a lower incidence of OHSS compared to GnRH agonist. $^{6}$

\section{Aspirin}

Increased platelet activation due to VEGF levels may lead to release of substances such as histamine, serotonin and platelet derived growth factor which can further potentiate the patho-physiological pathway of OHSS. Thus, aspirin has been considered for reduction of OHSS. ${ }^{7}$

\section{Metformin}

Metformin, an insulin sensitising drug, by improving intra ovarian hyper androgenism, metformin can affect the ovarian response by reducing the number of nonperiovulatory follicles. ${ }^{8}$

\section{Coasting}

Withholding gonadotropins at the end of $\mathrm{COH}$ for upto 4 days with an aim to reduce the risk of OHSS has also been postulated as a preventive strategy for OHSS.

\section{Choice of trigger for final oocyte maturation}

GnRH agonist as a trigger has been studied extensively and has been seen to eliminate OHSS in an antagonist cycle. Several RCTs have proven its preventive role in OHSS development and especially in a high risk population like PCOS women. ${ }^{9}$

\section{Dopamine agonist}

Since VEGF is the main vasoactive substance implicated in the pathophysiology of OHSS, VEGF antagonist in the form of a dopamine agonist may result in reduction of VEGF and thus the vascular permeability and other manifestations. ${ }^{10}$

\section{Albumin}

Albumin because of its inherent property of binding proteins increases the plasma oncotic pressure and counteracts the permeability of angiotensin II and thus may play a role in the prevention and management of OHSS. ${ }^{11}$

\section{Calcium gluconate infusion}

Increased calcium has been found to inhibit cAMP stimulated rennin secretion which decreases angiotensin II synthesis and finally prevents VEGF release breaking the pathway for OHSS occurrence. ${ }^{12}$

\section{Luteal phase GnRH antagonist}

GnRH antagonist was found to lower the VEGF concentrations in human granulosa lutein cell cultures as well as the expression of VEGF and VEGF-R in the hyper stimulated ovaries. ${ }^{13}$

\section{Cryopreservation of embryos}

A 'freeze all' technique or elective cryopreservation of all the embryos with an aim to transfer in the subsequent non stimulated cycles is adopted to prevent the endogenous hCG rise should a pregnancy happen and further exacerbate the late onset OHSS.

\section{CONCLUSION}

Our case shows the importance of early diagnosis in pregnant patients presenting with acute abdomen and ovarian masses, since spontaneous OHSS may be associated with life-threatening complications. The aetiology needs to be determined to focus the treatment and avoid future complications.

\section{Funding: No funding sources Conflict of interest: None declared \\ Ethical approval: Not required}

\section{REFERENCES}

1. Naredi N, Singh SK, Lele P, Nagraj N. Severe ovarian hyperstimulation syndrome: Can we eliminate it through a multipronged approach? MJAFI 2018;74(1):44-50.

2. Dey AK, Dubey A, Mittal K, Kale S. Spontaneous ovarian hyperstimulation syndrome - understanding the dilemma, Gynecol. Endocrinol. 2015;20(2015):13 .

3. Lee TH, Liu CH, Huang CC, Wu YL, Shih YT, Ho $\mathrm{HN}$, et al. Serum antimullerian hormone level and estradiol levels as predictors of ovarian hyperstimulation syndrome in assisted reproductive technology cycles. Hum reprod. 2008;23:160-7.

4. Jayaprakasan K, Herbert M, Moody E, Stewart JA, Murdoch AP. Estimating the risks of ovarian hyperstimulation syndrome (OHSS): implications for egg donation for research. Hum Fertil (Camb). 2007;10(3):183-7.

5. De Leener A, Montanelli L, Van Durme J, Chae H, Smits G, Vassart G, et al. Presence and absence of follicle-stimulating hormone receptor mutations provide some insights into spontaneous ovarian hyperstimulation syndrome physiopathology. J Clin Endocrinol Metab. 2006;91(2):555-62.

6. Toftager M, Bogstad, Bryndorf T, Løssl K, Roskær J, Holland $\mathrm{T}$, et al. Risk of severe ovarian hyperstimulation syndrome in $\mathrm{GnRH}$ antagonist versus GnRH agonist protocol: RCT including 1050 first IVF/ICSI cycles. Hum Reprod. 2016;31(6):125364. 
7. Várnagy A1, Bódis J, Mánfai Z, Wilhelm F, Busznyák C, Koppán M. Low-dose aspirin therapy to prevent ovarian hyperstimulation syndrome. Fertil Steril. 2010;93(7):2281-4

8. Palomba S1, Falbo A, Carrillo L, Villani MT. MET formin in High Responder Italian Group. Metformin reduces risk of ovarian hyperstimulation syndrome in patients with polycystic ovary syndrome during gonadotropin-stimulated in vitro fertilization cycles: a randomized, controlled trial. Fertil Steril. 2011;96(6):1384-90.

9. Engmann L, DiLuigi A, Schmidt D, Nulsen J, Maier $\mathrm{D}$, Benadiva $\mathrm{C}$. The use of gonadotropin-releasing hormone $(\mathrm{GnRH})$ agonist to induce oocyte maturation after cotreatment with GnRH antagonist in high-risk patients undergoing in vitro fertilization prevents the risk of ovarian hyperstimulation syndrome: a prospective randomized controlled study. Fertil Steril. 2008;89(1):84-91.

10. Alvarez C1, Martí-Bonmatí L, Novella-Maestre E, Sanz R, Gómez R, Fernández-Sánchez $M$ et al. Dopamine agonist cabergoline reduces hemoconcentration and ascites in hyperstimulated women undergoing assisted reproduction. J Clin Endocrinol Metab. 2007;92(8):2931-7.

11. Venetis CA, Kolibianakis EM, Toulis KA, Goulis DG, Papadimas I, Basil C. Tarlatzis BC. Intravenous albumin administration for the prevention of severe ovarian hyperstimulation syndrome: a systematic review and metaanalysis. Fertil Steril. 2011;95(1):188-96.

12. Naredi N, Karunakaran S. Calcium gluconate infusion is as effective as the vascular endothelial growth factor antagonist cabergoline for the prevention of ovarian hyperstimulation syndrome. JHRS. 2013;6(4):248-52.

13. Lainas TG, S fontouris IA, Zorzovilis IZ, Petsas GK, Lainas GT, Iliadis GS, et al. Management of severe OHSS using GnRH antagonist and blastocyst cryopreservation in PCOS patients treated with long protocol. Reprod Biomed Online. 2009;18(1):15-20.

Cite this article as: Sharma AK, Rastogi R, Patel K, Vivek KG. Ovarian hyper stimulation syndrome in a spontaneous singleton pregnancy: a case report. Int J Reprod Contracept Obstet Gynecol 2022;11:632-5. 